TRANSACTIONS OF THE

AMERICAN MATHEMATICAL SOCIETY

Volume 354, Number 8, Pages 3075-3084

S 0002-9947(02)02971-9

Article electronically published on April 1, 2002

\title{
ALGEBRAIC STRUCTURE IN THE LOOP SPACE HOMOLOGY BOCKSTEIN SPECTRAL SEQUENCE
}

\author{
JONATHAN A. SCOTT
}

\begin{abstract}
Let $X$ be a finite, $n$-dimensional, $r$-connected CW complex. We prove the following theorem:

If $p \geq n / r$ is an odd prime, then the loop space homology Bockstein spectral sequence modulo $p$ is a spectral sequence of universal enveloping algebras over differential graded Lie algebras.
\end{abstract}

\section{INTRODUCTION}

Let $\Omega X$ be the Moore loop space on a pointed topological space $X$. If $R$ is a subring of $\mathbf{Q}$, then $H_{*}(\Omega X ; R)$ has a natural Hopf algebra structure via composition of loops, as long as there is no torsion. The submodule $P \subset H_{*}(\Omega X ; R)$ of primitive elements is a graded Lie subalgebra; in [6], Milnor and Moore showed that if $R=\mathbf{Q}$ and $X$ is simply connected, then $H_{*}(\Omega X ; \mathbf{Q})$ is the universal enveloping algebra of $P$. In [5], Halperin established the same conclusion for $R \subset \mathbf{Q}$ when $X$ is a finite, simply-connected CW complex, provided that $H_{*}(\Omega X ; R)$ is torsion-free and the least non-invertible prime in $R$ is sufficiently large.

In the presence of torsion, the loop space homology algebra with coefficients in $R$ does not have a natural Hopf algebra structure. However, in [3] Browder showed that the Bockstein spectral sequence $H_{*}\left(\Omega X ; \mathbf{F}_{p}\right) \Rightarrow\left(H_{*}(\Omega X ; \mathbf{Z}) /\right.$ torsion $) \otimes \mathbf{F}_{p}$ is a spectral sequence of Hopf algebras. Halperin also proved in [5] that for large enough primes, $H_{*}\left(\Omega X ; \mathbf{F}_{p}\right)$ is the universal enveloping algebra of a graded Lie algebra. The present article establishes this for every term in the Bockstein spectral sequence.

Theorem 1. Let $X$ be a finite, $n$-dimensional, $q$-connected $C W$ complex $(q \geq 1)$. If $p$ is an odd prime and $p \geq n / q$, then each term in the mod $p$ homology Bockstein spectral sequence for $\Omega X$ is the universal enveloping algebra of a differential graded Lie algebra $\left(L^{r}, \beta^{r}\right)$. In addition, the $E^{\infty}$ term is the universal enveloping algebra of a graded Lie algebra.

Note that the association $X \rightsquigarrow\left\{\left(L^{r}, \beta^{r}\right)\right\}$ is not functorial; see Example 1 Remark 1. The hypothesis $p \geq n / q$ cannot be removed. Indeed, $X=\Sigma \mathbf{C} P^{p}$ is 2-connected, but $(2 p+1)$-dimensional. There is a non-vanishing $p$ th power in $H^{*}\left(\Omega X ; \mathbf{F}_{p}\right)$ and so $H_{*}\left(\Omega X ; \mathbf{F}_{p}\right)$ is not primitively generated (see [6] $)$. In particular, it is not a universal enveloping algebra. The Bockstein spectral sequence is constant, so none of the terms is a universal enveloping algebra.

Received by the editors November 1, 2001.

2000 Mathematics Subject Classification. Primary 55P35; Secondary 16S30.

Key words and phrases. Loop space homology, Bockstein spectral sequence, universal enveloping algebra. 
Remark 2. Theorem 1 neglects the case $p=2$. If $X$ is $n$-dimensional and $q$ connected with $n \leq 2 q$, then $X$ is in the stable range. Indeed, if $q=1$, then $X$ is a finite wedge of 2 -spheres. If $q \geq 2$, then $X=\Sigma^{2} Y$, for some connected space $Y$, and the adjunction map $\Sigma Y \rightarrow \Omega X$ determines Hopf algebra isomorphisms $T E_{+}^{r}(\Sigma Y) \stackrel{\cong}{\rightrightarrows} E^{r}(\Omega X)$ for $r \geq 1$. In either case, the Bockstein spectral sequence is a free associative algebra generated by primitive elements, and so is naturally the universal enveloping algebra of a free adjusted Lie algebra in the sense of Sjödin [7].

In 2], under the hypotheses of Theorem [1. Anick associates to $X$ a differential graded Lie algebra $L_{X}$ over $\mathbf{Z}_{(p)}$ and a natural quasi-isomorphism $U L_{X} \stackrel{\cong}{\rightrightarrows}$ $C_{*}\left(\Omega X ; \mathbf{Z}_{(p)}\right)$ of Hopf algebras up to homotopy. It follows that there is an isomorphism of $\bmod p$ Bockstein spectral sequences $E^{r}\left(U L_{X}\right) \cong E^{r}(\Omega X)$. The inclusion $\iota_{X}: L_{X} \hookrightarrow U L_{X}$ therefore induces a transformation of Bockstein spectral sequences $E^{r}\left(\iota_{X}\right): E^{r}\left(L_{X}\right) \rightarrow E^{r}(\Omega X)$.

Theorem 2. The image of each $E^{r}\left(\iota_{X}\right)$ is contained in $L^{r}$.

Given the results of [2], Theorems 1 and 2 follow immediately from the following purely algebraic result:

Theorem 3. Let $(L, \partial)$ be a differential graded Lie algebra over $\mathbf{Z}_{(p)}$ that is connected, free as a graded module, and of finite type. The mod $p$ homology Bockstein spectral sequence for $U(L, \partial)$ is a sequence of universal enveloping algebras, $E^{r}(U L)=U\left(L^{r}, \beta^{r}\right)$, and converges to the universal enveloping algebra of a graded Lie algebra $L^{\infty}$. Furthermore, if $\iota: L \hookrightarrow U L$ is the inclusion, then the image of $E^{r}(\iota)$ is contained in $L^{r}$.

The proof of Theorem [3 depends in an essential way on the work of André [1] and Sjödin [7], which characterizes the cocommutative Hopf algebras of finite type over a field $\boldsymbol{k}$ which can be written as universal enveloping algebras. Namely, such a Hopf algebra $A$ can be written as $U L$ if and only if the dual $A^{\sharp}$ can be given the structure of a Hopf algebra with divided powers (see Theorem 4).

The structure of the article is as follows.

Section [1. Notation and review of graded Lie algebras, divided powers algebras, Bockstein spectral sequences, acyclic closures and minimal models.

Section 2, In [5], Halperin showed that for a differential graded Lie algebra $(L, \partial)$ over $\mathbf{F}_{p}, H(U L)=U E$ for a graded Lie algebra $E$. We show that the inclusion $\iota:(L, \partial) \hookrightarrow U(L, \partial)$ satisfies $\operatorname{im} H(\iota) \subset E$.

Section 3. We extend André-Sjödin duality 1, 7, between graded Lie algebras and graded Hopf algebras with divided powers, to the respective differential categories.

Section 4 Proof of Theorem 3

Section 5. An example is given, demonstrating that the sequence of Lie algebras given by Theorem 3 is not natural.

Acknowledgments. This paper is the result of work for my Ph.D. thesis. I would like to take this opportunity to thank my Ph.D. advisor, Steve Halperin, who suggested the problem of studying torsion in loop space homology, and who patiently and consistently provided encouragement, deep insight, and support. 


\section{Preliminaries}

Let $R$ be a commutative ring containing $1 / 2$. All objects are graded by the integers unless otherwise stated. Fix an odd prime $p$. The ring of integers localized at $p$ is denoted $\mathbf{Z}_{(p)}$ while the prime field is denoted $\mathbf{F}_{p}$. Differential graded modules, algebras, coalgebras, and Hopf algebras are shortened to DGM, DGA, DGC, and DGH, respectively; a comprehensive treatment of these objects is given in [4].

1.1. Graded modules. Let $M$ be a graded module over $R$. If $x \in M_{k}$ then we say that $x$ has degree $k$, and write $|x|=k$. A free graded module $M$ is of finite type if each $M_{k}$ is of finite rank. We raise and lower degrees by the convention $M^{k}=M_{-k}$. We denote by $s M$ the suspension of $M:(s M)_{i}=M_{i-1}$. The dual of $M$ is the graded module $M^{\sharp}=\operatorname{Hom}(M, R)$. If $M$ is finite type and $N=(s M)^{\sharp}$, then $M=(s N)^{\sharp}$ via $x(s f)=-f(s x)$, for $x \in M, f \in N$.

If $V$ is a graded module over $R$, then we denote by $T V$ and $\Lambda V$ the tensor algebra and free commutative algebra on $V$, respectively. The tensor coalgebra on $V$ is denoted by $T_{C} V$. The shuffle product ([5], Appendix) makes $T_{C} V$ into a graded commutative (not cocommutative) Hopf algebra. Note that $T V=\bigoplus_{k \geq 0} T^{k} V$, $\Lambda V=\bigoplus_{k>0} \Lambda^{k} V$ and $T_{C} V=\bigoplus_{k>0} T_{C}^{k} V$, with $T^{k} V, \Lambda^{k} V$, and $T_{C}^{k} V$ consisting of words in $V$ of length $k$. Elements of $T_{C}^{k} V$ are denoted $\left[v_{1}|\cdots| v_{k}\right]$.

The symmetric group $S_{k}$ acts on $T^{k} V$ via $\sigma \cdot\left(x_{1} \otimes \cdots \otimes x_{k}\right)= \pm x_{\sigma(1)} \otimes \cdots \otimes x_{\sigma(k)}$, where the sign is determined by the rule $x \otimes y \mapsto(-1)^{|x||y|} y \otimes x$.

1.2. Graded Lie algebras. A graded Lie algebra is a graded $R$-module $L=$ $\bigoplus_{k \geq 0} L_{k}$ along with a degree-zero linear map [,] : $L \otimes L \rightarrow L$, called the Lie bracket, satisfying graded anti-symmetry, the graded Jacobi identity, and the further condition $[x,[x, x]]=0$ if $x \in L_{\text {odd }}$; see [5] for details.

For example, any non-negatively graded associative algebra $A$ is a graded Lie algebra via the graded commutator bracket $[a, b]=a b-(-1)^{|a||b|} b a$, for $a, b \in A$.

A graded Lie algebra is connected if it is concentrated in strictly positive degrees.

The graded abelian Lie algebra on the graded set $\left\{x_{j}\right\}$, denoted $L_{\mathrm{ab}}\left(x_{j}\right)$, is the free graded module on the basis $\left\{x_{j}\right\}$, given the trivial Lie bracket.

Let $L$ be a graded Lie algebra, and denote by $L^{b}$ the underlying graded module. The universal enveloping algebra of $L$ is the associative algebra $U L=\left(T L^{b}\right) / I$, where $I$ is the ideal generated by all elements of the form $x \otimes y-(-1)^{|x||y|} y \otimes x-$ $[x, y]$, for $x, y \in L$. $U L$ has the natural structure of a graded Hopf algebra; the comultiplication is defined by declaring the elements of $L$ to be primitive and then using the universal property.

A Lie derivation on a graded Lie algebra $L$ is a linear operator $\theta$ on $L$ of degree $k$ such that for $x, y \in L, \theta([x, y])=[\theta(x), y]+(-1)^{k|x|}[x, \theta(y)]$. A differential graded Lie algebra (DGL for short) is a pair $(L, \partial)$, where $L$ is a graded Lie algebra, and $\partial$ is a Lie derivation on $L$ of degree -1 satisfying $\partial \partial=0$. If $(L, \partial)$ is a DGL, then $\partial$ extends to a derivation on $U L$, making $U(L, \partial)$ into a DGA.

1.3. Divided powers algebras. Divided powers algebras arise as the duals of universal enveloping algebras, in the sense of Theorem 4 , below.

Definition 1. A divided powers algebra, or $\Gamma$-algebra, is a commutative graded algebra $A$, satisfying either $A=A^{\geq 0}$ or $A=A \leq 0$, equipped with set maps $\gamma^{k}$ : $A^{2 n} \rightarrow A^{2 n k}$ for $k \geq 0$ and $n \neq 0$ satisfying the following list of conditions. 
(1) $\gamma^{0}(a)=1 ; \gamma^{1}(a)=a$ for $a \in A$;

(2) $\gamma^{k}(a+b)=\sum_{j=0}^{k} \gamma^{j}(a) \gamma^{k-j}(b)$ for $a, b \in A^{2 n}$;

(3) $\gamma^{j}(a) \gamma^{k}(a)=\left(\begin{array}{c}j+k \\ j\end{array}\right) \gamma^{j+k}(a)$ for $a \in A^{2 n}$;

(4) $\gamma^{j}\left(\gamma^{k}(a)\right)=\frac{(j k) !}{k !^{j} j !} \gamma^{j k}(a)$ for $a \in A^{2 n}$;

(5) $\gamma^{k}(a b)= \begin{cases}a^{k} \gamma^{k}(b) & \text { if }|a| \text { and }|b| \text { even, }|b| \neq 0, \\ 0 & \text { if }|a| \text { and }|b| \text { odd. }\end{cases}$

A $\Gamma$-morphism is an algebra morphism which respects the divided powers operations. A Hopf $\Gamma$-algebra is a Hopf algebra, along with a system of divided powers, such that the coproduct is a $\Gamma$-morphism. A $\Gamma$-derivation on a $\Gamma$-algebra $A$ is a derivation $\theta$ on $A$ satisfying $\theta\left(\gamma^{k}(a)\right)=\theta(a) \gamma^{k-1}(a)$ for $a \in A^{2 n}, k \geq 1$. A differential graded $\Gamma$-algebra, or $\Gamma$-DGA, is a pair $(A, \partial)$, where $A$ is a $\Gamma$-algebra, and $\partial$ is a $\Gamma$-derivation of degree +1 satisfying $\partial \partial=0$. If $A$ is furthermore a Hopf $\Gamma$-algebra, and $\partial$ is also a coderivation, then $(A, \partial)$ is called a differential graded Hopf $\Gamma$-algebra, or $\Gamma$-DGH.

Let $V$ be a free graded $R$-module. Let $\Gamma^{k}(V)$ be the graded submodule of $T_{C}^{k} V$ of elements fixed by the action of the symmetric group $S_{k}$. Then $\Gamma(V)=\bigoplus_{k} \Gamma^{k}(V)$ is a Hopf subalgebra of $T_{C}(V)$, called the free $\Gamma$-algebra on $V$. Divided powers are defined on $\Gamma(V)$ by

(1) $\gamma^{0}(v)=1, \gamma^{1}(v)=v$ for $v \in V$,

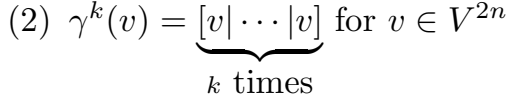

and then extending via conditions (4) and (5) of Definition 1 1 . If $f: V \rightarrow A$ is any linear map of degree zero from $V$ into a $\Gamma$-algebra $A$, then $f$ extends to a unique $\Gamma$-morphism $\bar{f}: \Gamma(V) \rightarrow A$. If $V$ is $R$-free on a countable, well-ordered basis $\left\{v_{i}\right\}$, then $\Gamma(V)$ is $R$-free, with basis consisting of elements $\gamma^{k_{1}}\left(v_{1}\right) \cdots \gamma^{k_{s}}\left(v_{s}\right)$ where $k_{j} \geq 0$ and $k_{j}=0$ or 1 if $\left|v_{j}\right|$ is odd.

Abusing notation, we will call a $\Gamma$-DGH free if it is free as a $\Gamma$-algebra.

Hopf $\Gamma$-algebras and universal enveloping algebras are related in the following sense.

Theorem 4 (André-Sjödin). Let $A$ be a connected Hopf algebra of finite type over a field $\boldsymbol{k}$, where char $\boldsymbol{k} \neq 2$. Then its dual Hopf algebra $A^{\sharp}$ is isomorphic to the universal enveloping algebra of a graded Lie algebra over $\boldsymbol{k}$ if and only if $A$ is a Hopf Г-algebra.

Remark 3. Theorem 4 was proved first by André in [1] in dual form. Sjödin proved the result directly in [7] and, using the notion of "adjusted" graded Lie algebras for characteristic 2, extended the result to arbitrary characteristic. Sjödin also proved for the "if" direction that $A$ is free as a $\Gamma$-algebra.

If $V \otimes W \stackrel{\langle,\rangle}{\rightarrow} R$ is a pairing, then there is an induced pairing

$$
T V \otimes T_{C} W \rightarrow R
$$

given by $\left\langle T^{j} V, T_{C}^{k} W\right\rangle=0$ if $j \neq k$, and

$$
\left\langle v_{1} \otimes \cdots \otimes v_{k},\left[w_{1}|\cdots| w_{k}\right]\right\rangle= \pm\left\langle v_{1}, w_{1}\right\rangle \cdots\left\langle v_{k}, w_{k}\right\rangle
$$


where \pm is the sign of the permutation $v_{1}, \ldots, v_{k}, w_{1}, \ldots, w_{k} \mapsto v_{1}, w_{1}, \ldots, v_{k}, w_{k}$. The pairing (1) in turn induces a pairing

$$
\Lambda V \otimes \Gamma W \rightarrow R .
$$

Suppose that $V$ is $R$-free of finite type, $V=V_{<0}$ or $V=V_{>0}$, and $W=V^{\sharp}$. Then (11) and (2) induce Hopf algebra isomorphisms $T_{C}\left(V^{\sharp}\right) \cong(T V)^{\sharp}$ and $\Gamma\left(V^{\sharp}\right) \cong(\Lambda V)^{\sharp}$.

1.4. The Cartan-Chevalley-Eilenberg-Cartan complex. Denote by $B(A)$ the bar construction on the augmented DGA $(A, \partial)$ ([5], Section 1); recall that the underlying coalgebra of $B(A)$ is $T_{C}(s \bar{A})$, where $\bar{A}$ is the augmentation ideal. Let $(L, \partial)$ be a DGL. Then $\Gamma(s L) \subset \Gamma(s \overline{U L}) \subset B(U L)$ and $\left(\Gamma(s L), \partial_{0}+\partial_{1}\right)$ is a sub-DGC of $B(U L)$, denoted by $C_{*}(L, \partial)$, called the chains on $(L, \partial)$.

The Cartan-Chevalley-Eilenberg-Cartan complex on $(L, \partial)$ is the commutative cochain algebra $C^{*}(L, \partial)=(\Lambda V, d)$, dual to $C_{*}(L, \partial)$ by the pairing (2), where $V=(s L)^{\sharp}$, and the differential $d$ is the sum of derivations $d_{0}$ and $d_{1}$. The linear part $d_{0}$ preserves word length and is dual to $\partial$ in that $\left\langle d_{0} v, s x\right\rangle=(-1)^{|v|}\langle v, s \partial x\rangle$ for $v \in V, x \in L$. The quadratic part $d_{1}$ increases word length by one and is dual to the Lie bracket in $L$ :

$$
\left\langle d_{1} v, s x \cdot s y\right\rangle=(-1)^{|s y|}\langle v, s[x, y]\rangle
$$

where the pairing is (2) above with $W=s L=V^{\sharp}$. We will usually refer to the Cartan-Chevalley-Eilenberg-Cartan complex as the cochains on $(L, \partial)$.

1.5. Bockstein spectral sequences. Fix a prime $p$. Let $C$ be a free chain complex over $\mathbf{Z}_{(p)}$. Applying $C \otimes-$ to the short exact sequence of coefficient modules $0 \rightarrow \mathbf{Z}_{(p)} \stackrel{\times p}{\rightarrow} \mathbf{Z}_{(p)} \rightarrow \mathbf{F}_{p} \rightarrow 0$ leads to a long exact sequence in homology which may be wrapped into the exact couple

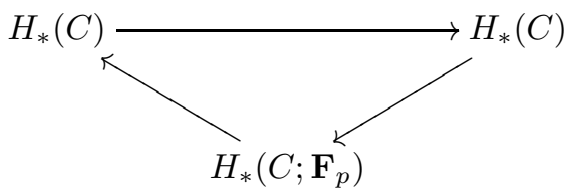

from which we get the homology Bockstein spectral sequence modulo $p$ of $C,\left(E^{r}(C)\right.$, $\left.\beta^{r}\right)$, mod $p$ BSS for short 3. If $C=C_{*}(X)$ is the normalized singular chain complex of a space $X$, then we refer to the homology BSS $\bmod p$ of $C_{*}(X)$ as the $\bmod p$ homology BSs of $X$, denoted $\left(E^{r}(X), \beta^{r}\right)$.

If $H(C)$ is of finite type, then the spectral sequence collapses degreewise to a graded vector space $E^{\infty}$, where $E^{\infty} \cong(H(C) /$ torsion $) \otimes \mathbf{F}_{p}$.

There is the corresponding notion of cohomology Bockstein spectral sequence defined in the obvious manner, using the functor $\operatorname{Hom}(C,-)$ rather than $C \otimes-$.

The $\bmod p$ Bss of $C$ measures $p$-torsion in $H_{*}(C)$ : if $x, y \in E^{r}, x \neq 0$, satisfy $\beta^{r}(y)=x$, then $x$ represents a torsion element of order $p^{r}$ in $H_{*}(C)$.

Notation. If $c \in C$ is such that $[\bar{c}] \in E^{1}$ lives until the $E^{r}$ term, then we will denote the corresponding element of $E^{r}$ by $[c]_{r}$.

1.6. Acyclic closures and minimal models. (Reference: [5, Sections 2 and 7]) Consider the graded algebra $\Lambda V \otimes \Gamma(s V)$ over $R$. Extend the divided powers operations on $\Gamma(s V)$ to $R \oplus \Lambda V \otimes \Gamma^{+}(s V)$ via rule 5 of Definition 1 
Definition 2 ([5, Section 2]). An acyclic closure of the DGA $(\Lambda V, d)$ is a DGA of the form $C=(\Lambda V \otimes \Gamma(s V), D)$ in which $D$ is a $\Gamma$-derivation restricting to $d$ in $\Lambda V$ and $H(C)=H^{0}(C)=R$.

Remark 4. Let $(L, \partial)$ be a connected DGL over $R$ which is $R$-free of finite type. Then $C^{*}(L)=(\Lambda V, d)$ where $V=(s L)^{\sharp}$. Let $C$ be an acyclic closure for $C^{*}(L)$, and set $(\Gamma(s V), \bar{D})=R \otimes_{C^{*}(L)} C$. By the work of Halperin in [5], we identify $H(U L)=H\left([\Gamma(s V), \bar{D}]^{\sharp}\right)$ and $U(L, \partial)=(\Gamma(s V), \bar{D})^{\sharp}$.

Let $R=\mathbf{Z}_{(p)}$ or $R=\mathbf{F}_{p}$, and consider a commutative algebra of the form $(\Lambda W, d)$ over $R$, where $W=W^{\geq 2}$ is $R$-free and of finite type. We may write the differential as a sum $d=\sum_{j \geq 0} d_{j}$ where $d_{j}$ raises wordlength by $j$.

Definition 3. If $R=\mathbf{Z}_{(p)}$, the DGA $(\Lambda W, d)$ above is $\mathbf{Z}_{(p)}$-minimal if $d_{0}: W \rightarrow$ $p W$. If $R=\mathbf{F}_{p},(\Lambda W, d)$ is $\mathbf{F}_{p}$-minimal if $d_{0}=0$.

Suppose $(A, \partial)$ is a cochain algebra satisfying $H^{0}(A)=R, H^{1}(A)=0, H^{2}(A)$ is $R$-free, and $H^{*}(A)$ is of finite type. Then by [5], Theorem 7.1, there exists a quasi-isomorphism $m:(\Lambda W, d) \stackrel{\simeq}{\rightarrow}(A, \partial)$ from an $R$-minimal algebra. This quasiisomorphism is called a minimal model.

Associated to an $\mathbf{F}_{p}$-minimal model $m:(\Lambda W, d) \stackrel{\simeq}{\rightrightarrows}(A, \partial)$ is its homotopy Lie algebra, $E$. As a graded vector space, $E=(s W)^{\sharp}$; the bracket is defined by the relation

$$
\langle w, s[x, y]\rangle=(-1)^{|s y|}\left\langle d_{1} w, s x \cdot s y\right\rangle
$$

for $w \in W, x, y \in E$.

\section{The image of $H(L) \rightarrow H(U L)$}

Let $(L, \partial)$ be a connected DGL over $\mathbf{F}_{p}$ of finite type. By [5], the choice of minimal model $m:(\Lambda W, d) \stackrel{\simeq}{\rightarrow} C^{*}(L)$ determines an isomorphism of graded Hopf algebras, $H(U L) \cong U E$, where $E$ is the homotopy Lie algebra of $m$.

Proposition 5. With the notation above, the image of $H(\iota): H(L) \rightarrow H(U L)$ lies in $E$.

Proof. It suffices to construct the following commutative diagram.

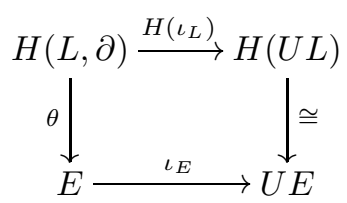

Recall that $C^{*}(L, \partial)=(\Lambda V, d)$, where $V=(s L)^{\sharp}$ and $d=d_{0}+d_{1}$. Recall further that the minimality condition on $(\Lambda W, d)$ implies that the linear part of its differential vanishes. The linear part of $m$ is the linear map $m_{0}:(W, 0) \rightarrow\left(V, d_{0}\right)$ defined by the condition $m-m_{0}: W \rightarrow \Lambda^{\geq 2} V$. Recall that $E=(s W)^{\sharp}$ and $U E=\Gamma(s W)^{\sharp}([5]$, Theorem 6.2).

The model $m$ extends to a morphism of constructible acyclic closures ([5], Section 2) $\hat{m}:(\Lambda W \otimes \Gamma(s W), D) \rightarrow(\Lambda V \otimes \Gamma(s V), D)$ by Proposition 2.7 of [5]. Since $(\Lambda W, d)$ is $\mathbf{F}_{p}$-minimal, $d_{0}=0$. By Corollary 2.6 of [5], $d_{0}=0$ is equivalent to $\bar{D}=0$ in $(\Gamma(s W), \bar{D})$. Apply $\mathbf{F}_{p} \otimes_{m}-$ to $\hat{m}$ to get a $\Gamma$-morphism $\bar{m}:(\Gamma(s W), 0) \rightarrow$ $(\Gamma(s V), \bar{D})$. 
Let $\pi_{L}:(\Gamma(s V), \bar{D}) \rightarrow s\left(V, d_{0}\right)$ and $\pi_{E}:(\Gamma(s W), 0) \rightarrow s(W, 0)$ be the projections. The maps $\pi_{L}$ and $\pi_{E}$ fit into the diagram

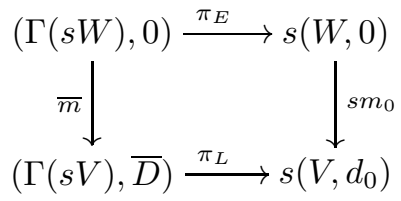

For $w \in W$, Proposition 2.7 of [5] states that $\hat{m}(1 \otimes s w)-1 \otimes s m_{0} w$ has total wordlength at least two. It follows that $\bar{m}(s w)-s m_{0} w$ has $\Gamma(s V)$-wordlength at least two, so $\pi_{L}(\bar{m}(s w))=s m_{0} w=s m_{0}\left(\pi_{E}(s w)\right)$, so diagram (5) commutes. Dualize and pass to homology to get (4).

\section{The dual of a $\Gamma$-Derivation}

André [1] and Sjödin [7] proved that the functor $L \rightsquigarrow(U L)^{\sharp}$ is a natural equivalence from connected graded Lie algebras of finite type over a field $\boldsymbol{k}$ to Hopf $\Gamma$-algebras of finite type over $\boldsymbol{k}$. In fact, by [7], $(U L)^{\sharp}=\Gamma(V)$, where $V^{\sharp}=L$ as a graded vector space. The same result is proved in [5] over an arbitrary commutative ring containing $1 / 2$. Let $R$ be a such a ring.

Proposition 6. Let $(U L, \partial)$ be a $\mathrm{DGH}$ over $R$ of finite type, where $L$ is a graded Lie algebra over $R$ which is free as an $R$-module. Then $\partial^{\sharp}$ is a $\Gamma$-derivation if and only if $\partial(L) \subset L$.

Proof. It suffices to prove the dual statement, namely that $\partial^{\sharp}: \Gamma V \rightarrow \Gamma V$ factors over the surjection $\pi: \Gamma V \rightarrow V$ to induce a differential in $V$. But $\operatorname{ker}(\pi)$ is generated as a module by products and elements of the form $\gamma^{k}(v)$ for $v \in V$, $k \geq 2$. Since $\partial^{\sharp}$ is a $\Gamma$-derivation, $\partial^{\sharp}\left(\gamma^{k}(v)\right)=\partial^{\sharp}(v) \gamma^{k-1}(v)$ is a product. It follows that $\partial^{\sharp}(\operatorname{ker}(\pi)) \subset \operatorname{ker}(\pi)$, completing the 'only if' portion of the proof.

Conversely, the work of Halperin in [5] allows us to identify $[U(L, \partial)]^{\sharp}$ with $\left(\Gamma\left(L^{\sharp}\right), \bar{D}\right)$ as DG Hopf algebras. Since $\bar{D}$ is a $\Gamma$-derivation, so too is $\partial^{\sharp}$.

We can thus extend the work of André and Sjödin to the differential categories.

Theorem 7. The functor $L \rightsquigarrow(U L)^{\sharp}$ is a natural equivalence, from the category of DGL's of finite type over $\boldsymbol{k}$ to the category of $\Gamma$-DGH's of finite type over $\boldsymbol{k}$.

\section{Bockstein SPECTRAL SEQUENCE OF A UNIVERSAL ENVELOPING AlGEBRA}

In this section, we prove the main algebraic result, Theorem 3, stated in the Introduction.

Let $(\Lambda W, d)$ be a minimal Sullivan algebra over $\mathbf{Z}_{(p)}$. Let $C=(\Lambda W \otimes \Gamma(s W), D)$ be a constructible acyclic closure for $(\Lambda W, d)([5]$, Section 2$)$. Let $(\Gamma(s W), \bar{D})$ be the quotient $\mathbf{Z}_{(p)} \otimes_{(\Lambda W, d)} C . C \otimes \mathbf{F}_{p}$ is a constructible acyclic closure for $(\Lambda W, d) \otimes \mathbf{F}_{p}$. Since $(\Lambda W, d)$ is $\mathbf{Z}_{(p)}$-minimal, $p$ divides $d_{0}$, so the linear part of the differential vanishes in $(\Lambda W, d) \otimes \mathbf{F}_{p}$. It follows by Corollary 2.6 of [5] that the differential in $(\Gamma(s W), \bar{D}) \otimes \mathbf{F}_{p}$ is null, so that $p$ divides $\bar{D}$. Set $E^{r}=E^{r}\left([\Gamma(s W), \bar{D}]^{\sharp}\right)$ and $E_{r}=E_{r}(\Gamma(s W), \bar{D})$. Let $\rho: \Gamma(s W) \rightarrow \Gamma(s W) \otimes \mathbf{F}_{p}=E_{1}$ be the reduction homomorphism.

Proposition 8. With the hypotheses and notation above, for $r \geq 1$, the following statements hold. 
(1) $\left(E_{r}, \beta_{r}\right)$ is isomorphic to a free $\Gamma$-DGH $\left(\Gamma\left(s W_{r}\right), \beta_{r}\right)$.

(2) There is a $\Gamma$-morphism $g_{r}: E_{r} \rightarrow E_{1}$ such that if $g_{r}(z)=\rho(a)$ for some $z \in E_{r}, a \in \Gamma(s W)$, then $z=[a]_{r}$.

(3) There is a graded Lie algebra $L^{r}$ such that $\left(E^{r}, \beta^{r}\right)=U\left(L^{r}, \beta^{r}\right)$ as a DGH. Furthermore, there exists a graded Lie algebra $L^{\infty}$ such that $E^{\infty}=U L^{\infty}$ as a Hopf algebra.

Proof. We proceed by induction. For $r=1$, let $W_{1}=W \otimes \mathbf{F}_{p}$. Since $p$ divides $\bar{D}, E_{1}=\Gamma\left(s W_{1}\right)$ and $\beta_{1}=\bar{D} / p$ (reduced modulo $p$ ). Because $\bar{D}$ is a $\Gamma$-derivation, so is $\beta_{1}$, establishing the first statement. For the second statement, let $g_{1}$ be the identity map on $E_{1}$. The third statement follows from the first and Theorem 7 In fact, from the definitions it follows that $L^{1}$ is the homotopy Lie algebra of the identity on $(\Lambda W, d) \otimes \mathbf{F}_{p}$.

Now suppose the three statements are established for $r-1$. We may write $C^{*}\left(L^{r-1}, \beta^{r-1}\right)=\left(\Lambda W_{r-1}, \delta\right)$; let $C(r-1)=\left(\Lambda W_{r-1} \otimes \Gamma\left(s W_{r-1}\right), D\right)$ be a constructible acyclic closure. By Lemma 5.4 of [5], there is a chain isomorphism $\gamma_{r-1}: U\left(L^{r-1}, \beta^{r-1}\right) \stackrel{\cong}{\longrightarrow}\left(\Gamma\left(s W_{r-1}\right), \bar{D}\right)^{\sharp}$. It is implicit that $\gamma_{r-1}$ is a coalgebra isomorphism, which then induces an algebra structure on $\left(\Gamma\left(s W_{r-1}\right), \bar{D}\right)^{\sharp}$ which makes $\left(\Gamma\left(s W_{r-1}\right), \bar{D}\right)$ into a $\Gamma$-DGH. In particular, under $\gamma_{r-1}$ we identify $\bar{D}$ with $\beta_{r-1}$, the differential in $E_{r-1}$.

Let $m_{r}:\left(\Lambda W_{r}, d\right) \stackrel{\simeq}{\rightarrow} C^{*}\left(L^{r-1}, \beta^{r-1}\right)$ be a minimal model. Let $C^{\prime}(r)$ be a constructible acyclic closure of $\left(\Lambda W_{r}, d\right)$ ([5], Section 2). Since $\left(\Lambda W_{r}, d\right)$ is $\mathbf{F}_{p}$-minimal, $d_{0}=0$; so by Corollary 2.6 of [5], $\mathbf{F}_{p} \otimes_{\Lambda W} C^{\prime}(r)=\left(\Gamma\left(s W_{r}\right), 0\right)$. By [5], Proposition 2.7, $m_{r}$ induces a $\Gamma$-morphism $\bar{m}_{r}:\left(\Gamma\left(s W_{r}\right), 0\right) \rightarrow\left(\Gamma\left(s W_{r-1}\right), \bar{D}\right)$. Since $\mathbf{F}_{p}$ is a field, by Lemma 3.3 of [5], we may identify $H\left(\bar{m}_{r}\right)$ with $\operatorname{Tor}^{m_{r}}\left(\mathbf{F}_{p}, \mathbf{F}_{p}\right)$, where Tor is the differential torsion functor [4]. Since $m_{r}$ is a quasi-isomorphism, $H\left(\bar{m}_{r}\right): \Gamma\left(s W_{r}\right) \stackrel{\cong}{\longrightarrow} H\left(\Gamma\left(s W_{r-1}\right), \beta_{r-1}\right)=E_{r}$, so $E_{r}$ is a free $\Gamma$-algebra. Furthermore, by [5], $H\left(\bar{m}_{r}^{\sharp}\right): E^{r}=H\left(U L^{r-1}\right) \stackrel{\cong}{\longrightarrow} U L^{r}$ as Hopf algebras, where $L^{r}=\left(s W_{r}\right)^{\sharp}$ as graded vector spaces. Therefore $E_{r}=\left(E^{r}\right)^{\sharp}$ is a Hopf $\Gamma$-algebra.

By the inductive hypothesis, there exists a $\Gamma$-morphism $g_{r-1}: E_{r-1} \rightarrow E_{1}$ such that $z=[a]_{r-1}$ whenever $z \in E_{r-1}, a \in \Gamma(s W)$ satisfy $g(z)=\rho(a)$. Let $g_{r}=g_{r-1} \bar{m}_{r}$. For $u \in E_{r}$ choose $a \in \Gamma(s W)$ so that $g_{r-1}\left(\bar{m}_{r}(u)\right)=\rho(a)$. Then $m_{r}(u)=[a]_{r-1}$, hence $\beta_{r-1}[a]_{r-1}=0$ and $[a]_{r} \in E_{r}$ is defined. Since $H\left(\bar{m}_{r}\right)[a]_{r}=$ $[a]_{r-1}, \bar{m}_{r}\left([a]_{r}\right)=[a]_{r-1}+\beta_{r-1}(v)$ for some $v \in E_{r-1}$. Thus $\bar{m}_{r}\left(u-[a]_{r}\right)=\beta_{r-1}(v)$, so $u-[a]_{r}$ is a boundary in $\left(E_{r}, 0\right)$, whence $u=[a]_{r}$. This establishes the second statement.

Let $u \in E_{r}$, and suppose for some $a \in \Gamma(s W)$ that $\rho(a)=g_{r}(u)$. Then $u=[a]_{r}$, so $\bar{D} a=p^{r} b$ for some $b \in \Gamma(s W)$. Thus $\beta_{r}(u)=[b]_{r}$. Since $g_{r}$ and $\rho$ are $\Gamma$ morphisms, $\rho\left(\gamma^{j}(a)\right)=g_{r}\left(\gamma^{j}(u)\right)$ so $\gamma^{j}(u)=\left[\gamma^{j}(a)\right]_{r}$. Furthermore, $\bar{D}\left(\gamma^{k}(a)\right)=$ $p^{r} b \cdot \gamma^{k-1}(a) ;$ so

$$
\beta_{r} \gamma^{k}(u)=\beta_{r}\left[\gamma^{k}(a)\right]_{r}=\left[b \cdot \gamma^{k-1}(a)\right]_{r}=[b]_{r}\left[\gamma^{k-1}(a)\right]_{r}=\beta_{r}(u) \cdot \gamma^{k-1}(u) .
$$

Therefore $\beta_{r}$ is a $\Gamma$-derivation, finally establishing the first statement.

By Proposition 6, we have established the third statement, completing the inductive step.

The $E^{\infty}$ term. It suffices to show that $E_{\infty}$ is a Hopf $\Gamma$-algebra. From the definitions it follows that $E_{\infty}$ is a Hopf algebra. We have a sequence of $\Gamma$-morphisms

$$
\cdots \rightarrow E_{r} \stackrel{\bar{m}_{r}}{\longrightarrow} E_{r-1} \stackrel{\bar{m}_{r-1}}{\longrightarrow} E_{r-2} \rightarrow \cdots \stackrel{\bar{m}_{2}}{\longrightarrow} E_{1} .
$$


Thus we may identify $E_{\infty}$ with $\varliminf^{\lim } E_{r}$, which is the subset of $\Pi_{r \geq 1} E_{r}$ consisting of sequences $\left(x_{r}\right)$ that satisfy $x_{r}=\bar{m}_{r+1}\left(x_{r+1}\right)$ for all $r \geq 1$. Since each $\bar{m}_{r}$ is a $\Gamma$-morphism, a well-defined system of divided powers on $E_{\infty}$ is given by $\gamma^{k}\left(\left(x_{r}\right)\right)=$ $\left(\gamma^{k}\left(x_{r}\right)\right)$. From the definitions, the coproduct is a $\Gamma$-morphism.

Proof of Theorem 3 . Let $m:(\Lambda W, d) \stackrel{\simeq}{\rightarrow} C^{*}(L, \partial)$ be a minimal model. Recall that the underlying algebra of $C^{*}(L, \partial)$ is $\Lambda V$, where $V=(s L)^{\sharp}$. Let $(\Lambda W \otimes \Gamma(s W), D)$ and $(\Lambda V \otimes \Gamma(s V), D)$ be constructible acyclic closures for $(\Lambda W, d)$ and $C^{*}(L, \partial)$, respectively. The model $m$ determines a $\Gamma$-morphism $\bar{m}:(\Gamma(s W), \bar{D}) \rightarrow(\Gamma(s V), \bar{D})$ where $H\left(\bar{m}^{\sharp}\right)$ is an isomorphism. The composition

$$
U(L, \partial) \stackrel{\cong}{\leftrightarrows}(\Gamma(s V), \bar{D})^{\sharp} \cong(\Gamma(s W), \bar{D})^{\sharp}
$$

induces an isomorphism of Bockstein spectral sequences, establishing the first statement.

The reduced minimal model $m \otimes \mathbf{F}_{p}:(\Lambda W, d) \otimes \mathbf{F}_{p} \stackrel{\simeq}{\rightarrow} C^{*}(L, \partial) \otimes \mathbf{F}_{p}$ has homotopy Lie algebra $L^{1}$, so by Proposition [5, im $E^{1}(\iota) \subset L^{1}$. Suppose that $\operatorname{im} E^{r-1}(\iota) \subset$ $L^{r-1}$. Let $\iota^{(r-1)}: L^{r-1} \hookrightarrow U L^{r-1}$ be the inclusion. Then $\operatorname{im} E^{r}(\iota) \subset \operatorname{im} H\left(\iota^{(r-1)}\right)$.

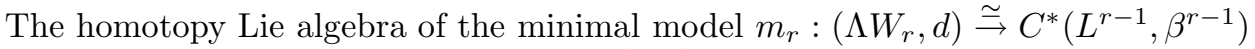
is $L^{r}$, so Proposition 5] states that im $H\left(\iota^{(r-1)}\right) \subset L^{r}$, completing the induction and the proof.

\section{EXAMPLE OF NON-NATURALITY}

First we state a proposition, whose proof is straightforward.

Proposition 9. Define a DGL over $\mathbf{F}_{p}$ by $(L, \partial)=\left(L_{a b}(e, f), \partial f=e\right)$, where $|f|=$ $2 n$. Then $C^{*}(L, \partial)=(\Lambda(x, y), d)$ with $d x=y$ and $|x|=2 n$. A minimal model $m$ : $\left(\Lambda\left(x_{1}, y_{1}\right), 0\right) \stackrel{\simeq}{\rightarrow} C^{*}(L, \partial)$, given by $x_{1} \mapsto x^{p}$ and $y_{1} \mapsto x^{p-1} y$, induces isomorphisms $\Gamma\left(s x_{1}, s y_{1}\right) \stackrel{\cong}{\rightrightarrows} H\left([U L]^{\sharp}\right)$ and $H(U L) \stackrel{\cong}{\rightrightarrows} U L_{a b}\left(e_{1}, f_{1}\right)$ with $\left|e_{1}\right|=\left|s x_{1}\right|=2 n p-1$, $\left|f_{1}\right|=\left|s y_{1}\right|=2 n p$.

Example 1. Define a DGL $(L, \partial)$ over $\mathbf{Z}_{(p)}$ by $L=L_{\mathrm{ab}}(e, f, g)$, where $|e|=2 n-1$, $|f|=|g|=2 n$, and $\partial(f)=p e$. Then $L^{1}=L_{\mathrm{ab}}(e, f, g)$ (over $\left.\mathbf{F}_{p}\right)$, with $\beta^{1}(f)=e$, and $C^{*}\left(L^{1}, \beta^{1}\right)=(\Lambda(x, y), d x=y) \otimes(\Lambda(z), 0)$. Recall the model $m$ from Proposition 9. Define DGA morphisms $i, j:(\Lambda(z), 0) \rightarrow C^{*}\left(L^{1}, \beta^{1}\right)$ by $i(z)=z, j(z)=z+y$. Then $\varphi=m \otimes i$ and $\psi=m \otimes j$ are minimal models, both with homotopy Lie algebra $L^{2}=L_{\mathrm{ab}}(a, b, c),|a|=2 n p-1,|b|=2 n p$, and $|c|=2 n$. The two models determine Hopf algebra isomorphisms $\varphi^{*}, \psi^{*}: H\left(U L^{1}\right) \rightarrow U L^{2}$, given by $\varphi^{*}\left[e f^{p-1}\right]=\psi^{*}\left[e f^{p-1}\right]=a, \varphi^{*}[g]=\psi^{*}[g]=c, \varphi^{*}\left[f^{p}\right]=b$, and $\psi^{*}\left[f^{p}\right]=b+c^{p}$. The algebra isomorphism $\psi^{*}\left(\varphi^{*}\right)^{-1}: U L^{2} \rightarrow U L^{2}$ is not of the form $U \theta$ for any Lie algebra morphism $\theta: L^{2} \rightarrow L^{2}$. Therefore the construction involved in Theorem [3 is not natural.

\section{REFERENCES}

[1] M. André, Hopf algebras with divided powers, J. Algebra 18 (1971), 19-50. MR 43:3323

[2] David J. Anick, Hopf algebras up to homotopy, J. Amer. Math. Soc. 2 (1989), no. 3, 417-453. MR 90c:16007

[3] William Browder, Torsion in H-spaces, Ann. of Math. (2) 74 (1961), 24-51. MR 23:A2201

[4] Y. Félix, S. Halperin, and J.-C. Thomas, Differential graded algebras in topology, Handbook of Algebraic Topology, North-Holland, Amsterdam, 1995, pp. 829-865. MR 96j:57052

[5] Stephen Halperin, Universal enveloping algebras and loop space homology, J. Pure Appl. Alg. 83 (1992), 237-282. MR 93k:55014 
[6] John W. Milnor and John C. Moore, On the structure of Hopf algebras, Ann. of Math. (2) 81 (1965), 211-264. MR 30:4259]

[7] Gunnar Sjödin, Hopf algebras and derivations, J. Algebra 64 (1980), 218-229. MR 84a:16016

Department of Mathematics, University of Toronto, Toronto, Ontario M5S 3G3, CANADA

Current address: Aberdeen Topology Centre, Department of Mathematical Sciences, University of Aberdeen, Aberdeen AB24 3UE, United Kingdom

E-mail address: j.scott@maths.abdn.ac.uk 